\title{
Ueber die theoretische Behandlung der sogenannten constanten Magnete*).
}

Von Carl Neumann in Leipzig.

Bei gegenseitiger Einwirkung zwischen magnetischen Körpern und galvanischen Strömen werden im Allgemeinen sämmtliche Ströme sich ändern, nicht nur die galvanischen Ströme selber, sondern auch die in jenen Körpern enthaltenen Molecularströme. Doch existiren gewisse Körper - die sogenannten constanten oder permanenten Stahlmagnete - , bei denen nan von der Aenderung der Molecularströme zu abstrahiren für zulässig erachtet. Denn man ist der Ansicht, dass die Molecularströme eines solchen Stahlmagneten approximativ als constant**) zu betrachten seien, hinsichtlich ihrer Stärke wie hiusichtlich ihrer Bahnen.

Ich werde dieser Ansicht mich vorläufig anschliessen. Demgemäss werde ich, um die gegenseitige (ponderomotorische und elektromotorische) Einwirkung zwischen galvanischen Strömen und sogenannten constanten Magneten durch bestimmte Forneln auszudrücken, einerseits vou den bekannten beiden Integralgesetzen, andrerseits von der Annabme constanter Molecularströme ausgehen; die in solcher Weise -sich ergebenden Formeln mögen kurzweg bezeichnet sein als die gewöhnlichen Formeln oder als die Formeln der gewöhnlichen Theorie. Sodann werde ich zeigen, dass diese Formeln zu Widersprüchen führen, und hieraus den Schluss ziehen, dass jene Annahme constanter Molecularströme theoretisch unzulässig sei.

*) Die nit der Jahrzahl 1871 versehenen Citate, welche im Laufe dieses Aufsatzes vorkommen, bezieben sich auf meine Schrift: Elektrodynamische Untersuchungen mit besonderer Rücksicht auf das Princip der Energie. Berichte d. Kgl. Sächs. Ges. d. Wiss., October 1871, S. 386-449.

**) Ich unterscheide zwischen' constant, gleichförmig und ungleichförmig. Eiu elektrischer Strom soll nämlich ungleichförmig heissen, wenn seine Stärke eine Function von Zeit und Bogenlänge ist; er soll gleichförmig genannt werden, wenn seine Stärke nur eine Function der Zeit ist, und er soll endlich constant genannt werden, wenn seiue Stärke weder von der Zeit, noch auch von der Bogenlänge abhängt. 
$\S 1$.

Die F. Neumann'schen Integralgesetze.

Es können diese Gesetze, welche in meinem bei Teubner erschienenem Werk: „Die elektrischen Kräfte" ausführlich erörtert worden sind, ihrem Hauptinhalte nach folgendermassen charakterisirt werden:

Das ponderomotorische Integralgesetz sagt aus, dass die von zwei gleichförmigen Stromringen während der Zeit dt auf einander ausgeïbte ponderomotorische Arbeit

$$
=-J J_{1} d Q
$$

ist, $200 \mathrm{~J}, J_{1}$ die beiden Stromintensitäten bezeichnen, während $Q[$ das sogenannte Potential, bezogen auf die Stromeinheit] einen Ausdruck vorstellt, der lediglich abhängt von der augenblicklichen Lage und Gestalt der beiden Ringe. - Vgl. „Die elektrischen Kräfte“, S. 55.

Das eleletromotorische Integralgesetz bezieht sich ebenfalls auf zwei gleichformige Stromringe, und sagt aus, dass die Summe der von dem einen Ringe während der Zeit dt im andern inducirten elektromotorischen Kräfte

$$
=+d\left(J_{1} Q\right)
$$

ist, wo $J_{1}$ die Stromintensität des inducivenden Ringes bezeichnet, während $Q$ dieselbe Bedeutung hat wie vorhin. - Vgl. "Die elektrischen Kräfter", S. 107.

\section{$\S 2$.}

Darstellung der gewöhnlichen Theorie.

Wir betrachtẹn zunäcbst ein System $\left(\boldsymbol{M}, \boldsymbol{M}_{1}\right)$, bestehend aus zwei constanten Stahlmagneten $M$ und $M_{1}$, welche sich bewegen unter ihrer gegenseitigen Einwirkung, sowie unter der Einwirkung beliebig gegebener äusserer Kräfte; dabei sei vorausgesetzt, dass diese äusseren Kräfte durchweg ordinärer (nichtelektrischer) Natur sind.

Bezeichnet man mit $j j_{1} Q$ das Potential eines Molecularstromes $j$ des Magneten $M$ auf einen Molecularstrom $j_{1}$ des Magneten $M_{1}$, so wird das Potential der beiden Magneten auf einander den Werth haben:

$$
\Sigma \Sigma\left(j j_{1} Q\right)
$$

die Summation ausgedehnt über alle $\ddot{j}$ und alle $j_{1}$. Das Potential $V$ des Systems $\left(\boldsymbol{M}, \boldsymbol{M}_{1}\right)$ auf sich selber wird daher dargestellt sein durch:

$$
V=\frac{1}{2} C+\frac{1}{2} C_{1}+\Sigma \Sigma\left(j j_{1} Q\right),
$$

wo $\frac{1}{2} C$ und $\frac{1}{2} C_{1}$ zwei Constante sind, von denen die erstere das Potential von $M$ auf sich selber, die letztere das Potential von $M_{1}$ auf sich selber vorstellt. 
Die ponderomotorische Arbeit, welche die Ströme $j$ und $j_{1}$ während der Zeit $d t$ auf einander ausüben, ist $=-j j_{1} d Q$, zufolge (1); und es wird daher die von den beiden Magneten auf einander ausgeübte Arbeit $=-\Sigma \Sigma\left(j j_{1} d Q\right)$ sein. Andrerseits mag die während der Zeit $d t$ von den äussern (ordinären) Kräften auf das System aus= geübte Arbeit benannt sein mit $d S$.

Bezeichnet nun $T$ die lebendige Kraft der ponderablen Massen des Systems, so ist bekanntlich ${ }^{-1 e r}$ dem Zeitelement $d t$ entsprechende Zuwachs $d T$ gleich der Summe sümmtlicher Arbeiten, welche während jenes Zeitelementes auf das System ausgeübt werden; somit erhält man:

$$
d T=-\Sigma \Sigma\left(j j_{1} d Q\right)+d S .
$$

Nun folgt aber aus (3), weil die $j, j_{1}$ constant und nur die $Q$ veränderlich sind, sofort: $a V=\Sigma \Sigma\left(j j_{1} d Q\right)$, so dass also die Formel (4) auch so geschrieben werden kann:

$$
d T=-d V+d S,
$$

in Worten ausgedrückt: Die während eines Zeitelementes erzeugte Quantität von lebendiger Kraft ist gleich dem negativen Zuwachs des Potentiales $V$, dazu gelegt die von den äussern Kräften ausgeübte Arbeit.

Wir gehen über zur Betrachtung zweier anderer Systeme, die wir kurzweg mit $\left(J, M_{1}\right)$ und $\left(J, J_{1}\right)$ benennen wollen.

Das System $\left(J, M_{1}\right)$ bestehe aus einem starren Ringe*), der von einem galvanischen Strome $J$ durchflossen ist, und aus einem constanten Stahlmagneten $M_{1}$. Die in dem Ringe ron Hause aus vorhandene elektromotorische Kraft mag mit $A$, sein Widerstand mit $w$ bezeichnet sein, so dass also, wenn der Ring sich selber überlassen wäre, nach dem $0 \mathrm{hm}$ 'schen Gesetz $J=\frac{A}{w}$ sein würde.

Fermer sei $j_{1}$ irgend einer der in $M_{1}$ enthaltenen Molecularströme und $J j_{1} Q$ das Potential von $J$ auf $j_{1}$, mithin

$$
J \cdot \Sigma\left(j_{1} Q\right)
$$

das Potential von $J$ anf $M_{1}$.

Das Potential $V$ des Systemes $\left(J, M_{1}\right)$ auf sich selber lautet alsdawn:

(6) $V=\frac{1}{2} q J^{2}+\frac{1}{2} C_{i}+J \cdot \Sigma(j, Q)$.
Das System $\left(J, J_{1}\right)$ bestehe aus zwei starren Ringen, die respective ron den galvanischen Strömen $J$ und $J_{1}$ durchflossen sind. Die in diesen Ringen von Hause aus vorhandenen elektromotorischen Kräfte mögen mit $A$ und $A_{1}$, ihre Widerstände mit $w$ und $w_{1}$ bezeichnet sein.
Ferner sei

$$
J J_{1} Q
$$

das Potential der beiden Ringe auf einander.

Das Potential $V$ des Systemes $\left(J, J_{1}\right)$ auf sich selber lautet alsdann:

$$
V=\frac{1}{2} q J^{2}+\frac{1}{2} q_{1} J_{1}^{2}+J J_{1} Q \text {. }
$$

*) Unter einem starren Ringe ist überall ein in sich zurücklaufender linearer Leiter von unveränderlicher Gestalt $z \alpha$ verstehen. 
Hier sind unter $\frac{1}{2} q J^{2}, \frac{1}{2} q_{1} J_{1}^{2}$ und $\frac{1}{2} C_{1}$ das Potential von $J$ auf sich selber, dasjenige von $J_{1}$ auf sich selber, und endlich dasjenige von $M_{1}$ auf sich selber za verstehen, so dass also $q, q_{1}$ und $C_{1}$ gewisse den beiden Ringen und dem Magneten eigenthümlich zugehörige Constanten repräsentiren.

Haben nun $d T$ und $d S$ analoge Bedeutungen wie vorhin [bei dem Systeme $\left.\left(\boldsymbol{M}, M_{1}\right)\right]$, so erhalten wir unter Anwendung des ponderomotorischen Integralgesetzes (1) die Formeln:

$$
\begin{aligned}
d T^{\prime} & =-J \cdot \Sigma\left(j_{1} d Q\right)+d S \\
& =-J \cdot d \Sigma\left(j_{1} Q\right)+d S ;
\end{aligned}
$$

es ist nämlich zu beachten, dass die $j_{1}$ zufolge unserer Annahme constant sind.

Andrerseits ergeben sich unter Benutzung des elektromotorischen Integralgesetzes (2) die Formeln:

(8) $w J d t=A d t+d\left[J q+\Sigma\left(j_{1} Q\right)\right]$,

$$
\begin{gathered}
w J d t=A d t+d\left(J_{q}+J_{1} Q\right), \\
w_{1} J_{1} d t=A_{1} d t+d\left(J_{1} q_{1}+J Q\right) ;
\end{gathered}
$$

und hieraus folgt durch Multiplication mit $J$ und $J_{1}$ sofort:

$$
\begin{aligned}
w J^{\mathrm{e}} d t=A J d t & +J \cdot d \Sigma\left(j_{1} Q\right) \\
& +d\left(\frac{1}{2} Q J^{2}\right),
\end{aligned} \quad \| \begin{aligned}
\left(w J^{2}+w_{1} J_{1}{ }^{2}\right) d t= & \left(A J+A_{1} J_{1}\right) d t+J J_{1} d Q \\
& +d^{2}\left(\frac{1}{2} q J^{2}+\frac{1}{2} q_{1} J_{1}^{2}+Q J J_{1}\right) .
\end{aligned}
$$

Endlich folgt durch Addition der Formeln (7) und (9) und mit Rücksicht auf $(6)$ :

$$
\begin{aligned}
& +w J^{2} d t= \\
& =A J d t+d\left(\frac{1}{2} q^{2}\right)+d S, \\
& \begin{array}{l}
d T+\left(w J^{2}+w_{1} J_{1}^{2}\right) d t \\
\quad=\left(A J+A_{1} J_{1}\right) d t+d V+d S
\end{array}
\end{aligned}
$$

§ 3.

Der in der gewöhnlichen Theorie enthaltene Widerspruch:

Die in (5) und (10) für die drei Systeme $\left(M, M_{1}\right),\left(J, \mathbb{M}_{1}\right)$ und $\left(J, J_{1}\right)$ erhaltenen Resultate sind von grosser Verschiedenheit, was besonders deutlich hervortritt, sobald man die Kräfte $A, A_{1}$ gleich Null annimmt. Denn alsdann nehmen jene Forneln (5) und (10) der Reihe nach folgende Gestalten an:

$$
\begin{aligned}
{[d T] } & =d S-d V, \\
{\left[d T+w J^{2} d t\right] } & =d S+d\left(\frac{1}{2} q J^{2}\right), \\
{\left[d T+w J^{2} d t+w_{1} J_{1}^{2} d t\right] } & =d S+d V,
\end{aligned}
$$

wo die in eckige Klammern [] eingesehlossenen Ausdrïcke dasjenige Quantum von lebendiger Kraft und Wärme repräsentiren, welches in jedem der drei Systeme während der Zeit dt erzeugt wird. Die Formeln sagen also aus, dass dieses Quantum für jene drei Systeme sehr verschiedene Werthe hat, dass z. B. dasselbe $=d S-d V$ oder 
$=d S+d V$ ist, je nachdem man das erste oder letate System betrachtet. - Die entwickelte Theorie steht also mit sich selber in Widerspruch.

Dieser Widerspruch ist ein sehr bedeutender; folglich sind die Prämissen der Theorie nicht nur im exacten, sondern auch im approximativen Sinne unzulässig. Jene Prämissen bestehen aber einerseits in den beiden Integralgesetzen (1), (2), andrerseits in der Annahme constanter Molecularströme; und man gelangt daher, falls man nicht etwa die beiden Integralgesetze in Frage stellen will, zu folgendem Resultat:

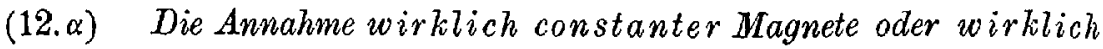
constanter Molecularströme ist theoretisch unzulässig, nicht nur im exacten, sondern auch im approximativen Sinne.

Demgemäss siud die mit dieser Annahme liirten Formeln $(3,4,5)$

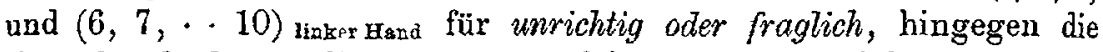
Formeln $(6,7, \ldots 10)$ rechter Hand, welche ron einer solchen Annahme frei sind, für zuverlässig zu halten.

Ist also irgend ein System. gegeben, welches theils aus sogenannten constanten Magneten, theils aus galvanischen Strömen besteht, und handelt es sich um eine nähere Untersuchung der in diesem System stattfindenden Wirkungen und Bewegungen, so wird eine solche Untersuchung, wie aus dem Satze (12. $\alpha$ ) unmittelbar folgt, als ein Problem höherer Ordnung zu bezeichnen sein, bei dessen sachgemässer Behandlung die Einfübrung irgend welcher Hypothesen über die innere $\mathrm{Me}-$ chanik magnetischer Körper nicht zu umgehen ist. .

So wird man z. B. bei einem derartigen System für das Princip der Erhaltung der Energie, bei sachgemässer Behandlung, eine Formel erhalten, die noch behaftet ist mit einer völlig unbekannten, von der innern Mechanik magnetischer Körper abhängenden Function; zur nähern Bestimmung dieser Function*) wird aber die Einführung irgend welcher Hypothesen über jene innere Mechanik unumgänglich erforderlich sein.

Aus (12. $\alpha)$ folgt also, dass das Gesetz der Erhaltung der Energie für solche Systeme, die aus elektrischen Strömen und sogenannten constanten Magneten bestehen, seiner eigentlichen Form nach einstweilen noch unbekannt ist.

Der Satz (12. $\alpha$ ) steht nicht isolirt da; es mag mir gestattet sein, auf verwandte Fälle aus bekannteren Regionen aufmerksam zu machen.

*) Der von mir (1871, S. 440 sq.) zmr ungefähren Bestimmung dieser Function gemachte Versuch ist, wie schon damals hetont wnrde, nur provisorisch und durch. aus unzureichend, 
Erster Fall. - Die Differentialgleichungen für einen frei beweglichen Massenpunkt $m$, auf welchen gegebene Kräfte $X, Y, Z$ einwirken, sind:

$$
m x^{\prime \prime}=X, \quad m y^{\prime \prime}=Y, \quad m z^{\prime \prime}=Z .
$$

Wollte man die Beweglichkeit des Punktes nachträglich noch durch eine gegebene Bedingung

$$
\varphi(x, y, z)=0
$$

beschränken, so würde manı vier Gleichungen $(\alpha),(\beta)$ haben, die mit einander in Widerspruch stehen. - In der That wird die Annahme oder Bedingung $\varphi(x, y, z)=0$ immer unzulässig sein, falls man nfcht gleichzeitig auch gewisse Kräfte*) hinzufügt, welche für Aufrechterhaltung jener Bedingung Sorge tragen.

Zweiter Fall. - Bewegen sich zwei Massenpunkte $m, M$ unter dem Einfluss ihrer gegenseitigen Anziehungs- oder Abstossungskraft längs einer gegebenen geraden Linie, und bezeichnet man ihre augenblicklichen Geschwindigkeiten mit $u, U$, so ergeben sich durch das Princip des Schwerpunkts und dureh das Princip der lebendigen Kraft die Formeln:

$$
\begin{gathered}
m u+M U=K, \\
m u^{2}+M U^{2}=F(r),
\end{gathered}
$$

wo $K$ eine Constante, hingegen $F(r)$ eine Function der gegenseitigen Entfernung $r$ der beiden Punkte vorstellt Wollte man zu diesen Formeln noch die Bedingung hinzutreten lassen:

$$
\bar{U}=\text { Const., }
$$

so würde ein Widerspruch eintreten; denn die Formel $(\gamma)$ würde alsdann aussagen, dass $u$ constant, die Formel $(\delta)$ hingegen, dass $u v a$ riabel sei. - Jene Annahme oder Bedingung $U=$ Const. ist also unzulässig.

Dritter Fall. - Die Massen $m$ und $M$ der soeben betrachteten Punkte mögen sich zu einander verhalten, wie die Masse eines Staubkorns zur Sonnenmasse. Offenbar wird alsdann die Sonnenmasse $\boldsymbol{M}$ (durch jenes Staubkorn $m$ so gut wie gar nicht afficirt) mit constanter oder wenigstens nahezu constanter Geschwindigkeit dahinfahren; so dass also die Annahme $(\varepsilon)$ wenigstens approximativ als zulässig er-scheint. - Aber der Schein trügt; denn durch Combinirung von $(\varepsilon)$ und $(\gamma),(\delta)$ würde man wiederum zu dem schon genannten Widerspruch, nämlieh zu dem Resultat geführt werden, dass die Geschwindigkeit $u$ des Staubkornes gleichzeitig constant und variabel sei. - Jene Annahme

*) Nämlich die bekannten Drnckkräfte $\lambda \frac{\partial \varphi}{\partial x}, \lambda \frac{\partial \varphi}{\partial y}, \lambda \frac{\partial \varphi}{\partial z}$. 
$U=$ Const. wird also im Allgemeinen unzulässig bleiben, wie gross auch $M$ gegen $m$ sein mag.

Aus diesen Betrachtungen ${ }^{*}$ ) dürfte sich die allgemeine Regel ergeben:

Soll die Bewegung gegebener Massen einer bestimmten Bedingungsgleichung unterworfen gedacht werden, so ist (falls innere Widersprïche vermieden werden sollen) die Einführung von Kräften erforderlich, welche für die Aufrechterhaltung jener Bedingungsgleichung Sorge tragen.

Will man also die elektrischen Molecularströme im Innern eines gegebenen Magneten als constant sich vorstellen, so ist man genöthigt, gleichzeitig auch gewisse Kräfte sich vorzustellen, welche für die Aufrechterhaltung dieser Constanz Sorge tragen.

Will man ferner (um ein anderes Beispiel anzuführen) die Vorstellung adoptiren, dass in einem elektrischen Strom jederzeit gleich grosse Quantitäten der beiden Fluida in entgegengesetzten Richtungen durch den Querschnitt gehen, so wird man gezwungen sein, gleichzeitig auch gewisse Kräfte zu adoptiren, welche für ein solches Gleichsein Sorge tragen.

\section{\$ 4.}

Zusammenstellung einiger Formeln der gewöhnlichen Theorie.

Wir beschränken uns auf die beiden Systeme

$$
\left(J, M_{1}\right), \quad \| \quad\left(J, J_{1}\right) \text {. }
$$

Das Potential $V(6)$ eines solchen Systemes auf sich selber besitzt den Werth :

(14) $V=\frac{1}{2} q J^{2}+\frac{1}{2} C_{1}+J \cdot \Sigma\left(j_{1} Q\right)$,

$$
V=\frac{1}{2} q^{2}+\frac{1}{2} q_{1} J_{1}^{2}+Q J J_{1}
$$

Die Formeln für die ponderomotorischen Wirkungen (7) lauten:

(15) $d T=-J \cdot d \Sigma\left(j_{1} Q\right)+d S$,

$$
d T=-J J_{1} d Q+d S
$$

diesen Formeln zufolge zerfällt die während der Zeit $d t$ im Systeme orzengte lebendige Kraft $d T$ in zwei Theile, nämlich in einen Theil

$$
-J \cdot d \Sigma\left(j_{1} Q\right) \text {, }
$$$$
-J J_{1} d Q \text {, }
$$

welcher speciell durch die elektrodynamischen, und in einen andern Theil

$$
d S \text {, }
$$

$d S$,

welcher speciell durch die ordinären Kräfte hervorgebracht wird.

Endlich sind für die elektromotorischen oder inducirenden Wirkungen entweder anzugeben die Formeln (9):

*) Aehnliche und zum Theil weiter ausgeführte Betrachtungen findet man in meiner Abhandlung (1871, S. 440-446). 


$$
\begin{aligned}
& A J d t=w J^{2} d t-J \cdot d \Sigma\left(j_{1} Q\right) \\
& -d\left(\frac{1}{2} q J^{2}\right) \text {. } \\
& \begin{aligned}
\left(A J+A_{1} J_{1}\right) d t & =\left(w J^{2}+w_{1} J_{1}{ }^{2}\right) d t-J J_{1} d Q \\
& -d\left(\frac{1}{2} q J^{2}+\frac{1}{2} q_{1} J_{1}{ }^{2}+Q J J_{1}\right) .
\end{aligned}
\end{aligned}
$$

oder auch die (diesen als Grundlage dienenden) detaillirteren Formeln (8): $\left(16^{\mathrm{bis}}\right) w J d t=A d t+d\left[J_{q}+\Sigma\left(j_{1} Q\right],\left\{\begin{array}{r}w J d t=A d t+d\left[J_{q}+J_{1} Q\right], \\ w_{1} J_{1} d t=A_{1} d t+d\left[J_{1} q_{1}+J Q\right] .\end{array}\right.\right.$

Diese Formeln $\left(16,16^{\text {bis }}\right)$ werden kurzweg zu bezeichnen sein als die Repräsentanten des gewöhnlichen Inductionsgesetzes.

Von Neuem mag, was schon bei Gelegenheit des Satzes $(12 . \alpha)$ bemerkt ist, wiederholt werden, dass die mit der Annahme constanter Molecularströme liirten Formeln $\left(14,15,16^{2}, 16^{\text {bis }}\right)_{1 \text { inker Hand }}$ als unzuverlässig, hingegen die Formeln $\left(14,15,16,16^{\text {tis }}\right)_{\text {rechter Hand }}$ als zuverlässig anzusehen sind.

\section{$\S 5$.}

\section{Das Princip der lebendigen Kraft.}

Ausgehend rom Weber'schen Grandgesetz und von einer gewissen unitarischen Vorstellungsweise, bin ich in meiner Abhandlung von $1871 \mathrm{zu}$ dem Resultat gelangt, dass das Princip der lebendigen Kraft in Gebiet der elektrischen Vorgänge repräsentirt ist durch zwei wohl von einander zu unterscheidende Sätze*), welche ich das Ener(fiegesetz und das Potentialgesetz benannte. Es mag mir gestattet sein, diese Sütze in etwas erweiterter Fassung kurz mitzutheilen**), sodann aber dieselben in Anwendung zu bringen auf die im Vorhergehenden betrachteten Systeme $\left(J, M_{1}\right)$ und $\left(J, J_{1}\right)$.

Ein System (linearer oder körperlicher) Conductoren sei in beliebiger Bewegung begriffen, während gleichzeitig im Innern eines jeden solchen Conductors irgend welche elektrische Yorgänge stattfinden; dabei mag vorausgesetzt sein, dass die auf das System von Aussen her einwirkenden Kräfte durchweg ordinärer (nicht elektrischer) Natur sind. Ferner sei $T$ die actuelle und $F$ die potentielle Energie des Systemes; mit andern Worten: es sei $T$ die lebendige Kraft aller in dem Systeme enthaltenen ponderablen Massen und es repräsentire $F$ den Ausdruck

$$
F=U^{o}+U-V
$$

*) Das Energiegesetz findet sich daselbst angegeben auf S. 408 und S. 414; andererseits das Potentialgesetz auf S. 430. Letzterer Name dürfte unzweckmüssig gewählt sein; ich habe daher später (z. B. in meinem Werk: ,Die elektrischen Kräfte") dieses letztere als ponderomotorisches Integralgesetz bezeichnet.

**) Eine ausführlichere Mittheilung der betreffenden Untersuchungen behalte ich mir vor. Hiebei wird sich alsdann Gelegenheit bieten zu erörtern, ob die yon Herm Helmboltz in seinem Aufsatz (1872, 8. 55, Note) ausgesprochene Behauptung, jene Untersuchungen seien mit einem mathematischen Fehler behaftet, begründet ist oder nicht. 
wo $U^{\circ}$ das ordinäre, $U$ das elektrostatische und $V$ das elektrodynamische Potential des Systemes auf sich selber bezeichnet. - Das Princip der lebendigen $\bar{K}$ raft ist alsdann repräsentirt durch folgende zwei Sätze:

Das Energiegesetz. Für jedes Zeitelement dt ist

$$
\begin{aligned}
d(T+F) & =d S-d Q^{*}, o d e r \\
d T+d\left(U^{\circ}+U-V\right) & =d S-d Q^{*},
\end{aligned}
$$

wo aS die während der Zeit dt von den äusseren Kräften verrichtete Arbeit, $\bullet$ und $d Q^{*}$ die während dieser Zeit (durch die elektrischen Vorgänge) im System entwickelte Wärme vorstellt*):

Das Potentialgesetz. Für jedes Zeitelement dt ist:

$$
a T+\delta\left(U^{\circ}+U+V\right)=d S,
$$

wo dS die schon genannte Bedeutung hat. Dabei ist unter $\delta\left(U^{\circ}+U+V\right)$ derjenige virtuelle Zuwachs zu verstehen, welchen die Grösse $\left(U^{\circ}+U+V\right)$ während der Zeit dt erlitten haben würde, falls während dieser Zeit die elektrischen Verhältnisse im Innern eines jeden Conductors ungeändert geblieben wären. - Zu bemerken ist, dass dieses Potentialgesetz nur dann gilt, wenn die elektrischen Strömungen im Innern der einzelnen Conductoren als gleichförmig und an ihren Oberflächen als tangential betrachtet werden dürfen.

Vernachlässigt man die durch $U^{\circ}$ und $U$ repräsentirten ordinären und elektrostatischen Kräfte gegenüber den durch $V$ repräsentirten elektrodynamischen Kräften, so können die Formeln (17) und (18. E, P) folgendermassen dargestellt werden:

$$
\begin{aligned}
F & =-V, \\
d T+d F & =d S-d Q^{*}, \\
d T-\delta F & =d S
\end{aligned}
$$

woraus durch Subtraction folgt:

$$
d F+\delta F=-d Q^{*}
$$

In meiner erwähnten Abhandlung ist der Werth der entwickelten Wärmemenge $d Q^{*}$ näher zu bestimmen versucht worden für den speciellen Fall linearer in sich zurücklaufender Conductoren; dabei ergab sich folgender Ausdruck**):

*) Ich bezeichne die entwickelte Wärmemenge mit $d Q^{*}$, um in solcher Weise Verwechselungen zu vermeiden. Denn der Buchstabe $Q$ (ohne Sternchen) ist bereits in anderer Bedeutung [vgl. $z_{m}$ B. Formel (1)] verwendet worden, und soll in dieser Bedeutung auch weiterhin beibehalten werden.

**) 1871 , S. 415 . 
(21) $d Q^{*}=\left(w J^{2}+w_{1} J_{1}^{2}+\cdots\right) d t-\left(A J+A_{1} J_{1}+\cdots\right) d t$,

wo $w, w_{1}, \cdots$ die Widerstände der einzeluen linearen Ringe, $J, J_{1}, \cdots$ die augenblicklich in ihnen vorhandenen Stromintensitäten, und $A$, $A_{1}, \ldots$ die in denselben ron Hause aus vorhandenen elektromotorischen Kräfte vorstellen.

Wir wollen nun diese Formeln in Anwendung bringen auf die in den vorhergehenden Paragraphen betrachteter Systeme

$$
\left(J, M_{1}\right), \quad \| \quad\left\langle J, J_{1}\right) \text {. }
$$

Für die potentielle Energie $F^{\prime}(19)$ eines solchen Systemes ergiebt sich, mit Rücksicht auf (14), folgender Werth:

$$
\begin{aligned}
F & =-V \\
& =-\left[\frac{2}{2} q J^{2}+\frac{2}{2} C_{1}+J \cdot \Sigma\left(j_{1} Q\right)\right],
\end{aligned}
$$

hieraus folgt:

$$
\delta F=-J \cdot \Sigma\left(j_{1} d Q\right),
$$

oder weil die $j_{1}$ unveränderlich sind:

$$
\delta F=-J \cdot d \Sigma\left(j_{1} Q\right) \text {. }
$$

$$
\begin{aligned}
F & =-V, \\
& =-\left[\frac{1}{2} q J^{2}+\frac{1}{2} q_{1} J_{1}^{2}+Q J J_{1}\right],
\end{aligned}
$$

hièraus folgt:

Ferner nimmt der Ausdruck der entwickelten Wärmemenge $d Q^{*}(21)$ für die betrachteten beiden Systeme folgende Gestalten an:

$$
d Q^{*}=w J^{2} d t-A J d t,
$$$$
\| d Q^{*}=\left(w J^{2}+w_{1} J_{1}^{2}\right) d t-\left(A J+A_{1} J_{1}\right) d t .
$$

Substituirt man nun die Werthe (23) und (24) in die allgemeine Formel $(20 . \mathbb{E}-\mathrm{P})$, so folgt:

$$
\begin{aligned}
& d F-J \cdot d \Sigma\left(j_{1} Q\right)= \\
& \quad=A J d t-w J^{2} d t ;
\end{aligned}
$$

hiefür kann geschrieben werden:

$$
\begin{gathered}
A J d t=w J^{2} d t-J \cdot d \Sigma(j, Q) \\
+d F,
\end{gathered}
$$

oder, falls man für $\boldsymbol{F}$ seine eigentliche Bedeutung (22) substituirt and durch $f$ dividirt:

$$
\text { (26 bis) } \begin{aligned}
w J d t=A d t & +d\left[q J+2 \Sigma\left(j_{1} Q\right)\right] \\
& +(d \log J) \cdot \Sigma\left(j_{1} Q\right) .
\end{aligned}
$$

$$
\begin{aligned}
& d F-J J_{1} d Q= \\
& =\left(A J+A_{1} J_{1}\right) d t-\left(w J^{2}+w_{1} J_{1}^{2}\right) d t_{;}
\end{aligned}
$$

hiefür kann geschrieben werden:

$$
\begin{gathered}
\left(A J+A_{1} J_{1}\right) d t=\left(w J^{2}+w_{1} J_{1}^{2}\right) d t-J J_{1} d Q \\
+d F .
\end{gathered}
$$

Diese Formel steht, falls man für $F$ seine eigentliche Bedeutung (23) substituirt, in vollem Einklang mit dem gewöhnlichen Inductionsgesetz $\left(16,16^{\text {bis }}\right)$.

Diese Formel $(26,26$ bis $)$ ist eine wildfremde Formel, welche von dem gewöhnlichen Inductionsgesetz $\left(16,16^{\text {bis }}\right)$ in greller Weise sich unterscheidet.

Die Formeln linker Hand sind also mit denen der gewöhnlichen Theorie in Widerspruch, während die rechter Hand mit denen der gewöhnlichen Theorie in Einklang stehen. Der Grund jenes Widerspruchs kann nur darin zu suchen sein, 
(27) dass constante Magnete nicht existiren und nicht existiren können; ebenso wenig etwa, wie ein Weltkörper gedacht werden kann, der trotz der Einwirkung der übrigen Weltkörper in geradliniger Bahn dahinfährt. Ein sogenannter constanter Magnet wird, falls er auf einen elektrischen Strom einwirkt, auch seinerseits von diesem Strome her eine gewisse Einwirkung und in seinem Zustande eine gewisse Aenderung erleiden. Diese $\mathrm{Zu}-$ standsänderung aber ist in unsern Formeln unberïcksichtigt gelassen.

In dieser Weise habe ich bereits in meiner Abhandlung von 1871 (daselbst S. 440) mich ausgedrückt*). Schon damals also war ich zu denjenigen Vorstellungen hingeleitet worden, die ich in den vorhergehenden Paragraphen des gegenwärtigen Aufsatzes von einer etwas anderen Seite her zu entwickeln und in $(12 . \alpha, \beta)$ möglichsit präcise auszusprechen mich bemüht habe.

In seiner bekannten Schrift über die Erhaltung der Kraft (Berlin 1847) hat $\mathrm{Helmholtz}$ die Formeln**) gegeben:

$$
A J d t=w J^{2} d t-J \cdot d \Sigma\left(j_{1} Q\right), \|\left(A J+A_{1} J_{1}\right) d t=\left(w J^{2}+w_{1} J_{1}^{2}\right) d t-J J_{1} d Q .
$$

Diese Helmholtz'schen Formeln habe ich in meiner Abhandlung (1871, S. 436, Note und S. 439, Note) für unzulässig erklärt, d. h. für unzulässig erklärt im Sinne der von mir entwichelten Theorie. In der That zeigt sich, dass den Helmholtz'schen Formeln (28) in Sinne meiner Theorie die Correctionen:

$d F$,

$$
\|
$$$$
d \boldsymbol{F},
$$

oder, falls man für $F$ seinen Werth (22) substituirt, die Correctionen:

$$
\text { (29. } \beta) d\left[-\frac{1}{2} q J^{2}-\frac{1}{2} C_{1}-J \cdot \Sigma\left(j_{1} Q\right)\right]
$$$$
d\left[-\frac{1}{2} q J^{2}-\frac{1}{2} q_{1} J_{1}^{2}-Q J J_{1}\right]
$$

zuzuertheilen sind. Mit andern Worten: Es zeigt sich, dass den Helmholtz'schen' Formeln (28) die genannten Correctionen (29, $\alpha, \beta)$. beizufügen sein $w \ddot{u} r d e n$, falls meine eigenell Formeln (26) die richtigen wären. Dass letzteres unwahrscheinlich ist, dass nämlich jene von mir selber gegebenen Formeln (26), wenigstens in soweit sie den Fall der Magnetoinduction betreffen, in grellem Widerspruch stehen

*) In jener Abhandlung sind übrigens auch die Formeln $(26,26$ bis ) in genau derselben Weise angegeben. Man findet die Formeln (26) linker Hand und (26) rechtex Hand respective auf $\mathrm{S} .439, \mathrm{Nr}$. (V.) und auf $\mathrm{S} .436, \mathrm{Nr}$. (II.), Ferner findet man die Formel (26 ${ }^{\text {bis }}$ ) auf $\mathrm{S}$. $440, \mathrm{Nr}$. (75).

**) Dass die Formeln der Helmboltz'schen Theorie von 1847 in der That durch (28) dargestellt sind, wird aus meinem unmittelbar folgenden Aufsatz (\$. 342) deutlich werden. 
mit dem gewöhnlichen Inductionsgesetz, ist vor wenig Augenblicken betont worden.

(30) Demgemäss sind also die in meiner Abhandlung von 1871 für die Helmholtz'schen Formelı von 1847 angemerkten Correctionen $(29 \alpha, \beta)$ nur von relativer Bedeutung, nämlich von solcher Beschaffenheit, dass sie jene Formeln allerdings in Einklang bringen würden mit der von mir daselbst entwickelten Theorie, andererseits aber dieselben in Widerspruch versetzen würden mit dem gewöhnlichen Inductionsgesetz.

Diesen nur relativen Charakter meiner Correctionen glaubte ich in meiner Abhandlung (1871) nicht besonders betonen zu dürfen, da derselbe unmittelbar aus dem Zusammenhange sich ergiebt. Denn der Gang meiner dortigen Erörterungen ist (um die Hauptsache zusammenzufassen) folgender:

Gestiitat auf bestimmte Suppositionen, nämlich auf das Princip der lebendigen Kraft und auf die Annahme constanter Magnete, gelange ich fiir die Magneto- und Volta-Induction zu den beiden Formeln (26), welche verschieden sind von den Helmholtz'schen Formdn (28), whe notire im Vorïbergehen die hieraus für die letztern sich ergebenden Correctionen $(29 . \alpha, \beta)$.

Sodann aber stellt sich heraus, dass meine eignen Formeln (26) mit den gewöhnlichen und für vichtig geltenden Gesetzen $\left(16,16^{\text {bis }}\right)$ theilweise in Widerspruch stehen; ein solcher Widerspruch zeigt sich bei der Magneto-Induction, während allerdings Einklang vorhanden ist bei der Volta-Induction.

Durch diesen Widerspruch sehe ich mich schliesslich in die Nothwendigkeit versetzt, einen gewissen Bestandtheil der zu Grumde gelegten Suppositionen fallen zu lassen; nämlich die Vorstellung constanter Magnete für unzulässig zu erklären; und gelange in solcher Weise zu den in (27) und (12. $\alpha, \beta)$ genannten Sötzen. 